\title{
ON THE TOPOGRAPHIC RECTIFICATION OF OCEAN FLUCTUATIONS
}

\author{
by
}

Alberto Álvarez, Emilio Hernández-García, Joaquín Tintoré

Instituto Mediterráneo de Estudios Avanzados IMEDEA ${ }^{1}$

CSIC-Universitat de les Illes Balears, E-07071 Palma de Mallorca (Spain).

\begin{abstract}
Stochastic fluctuations acting on a model of quasigeostrophic fluid motion on a rotating frame are shown to be rectified giving rise to large-scale noise-sustained average currents. As in other noise rectification phenomena, the effect requires nonlinearity and absence of detailed balance to occur. We apply an analytical coarse-graining procedure to obtain insight into the phenomenon. Relevance of the effect in the context of ocean modeling is briefly discussed.
\end{abstract}

\section{Introduction}

Nonlinear interactions can rectify random inputs of energy organizing them into coherent motion. This noise-rectification phenomenon has been discussed in several contexts ranging from biology to physics or engineering [1]. Three ingredients are needed to obtain this kind of noise-sustained directed motion: nonlinearity, random noise lacking the property of detailed balance, and some symmetry-breaking feature establishing a preferred direction of motion.

It has been shown numerically [2] that directed motion sustained by noise also appears in a model of large-scale fluid dynamics on a rotating frame, namely the vorticity equation describing quasigeostrophic forced turbulence [3]. A large amount of rotating fluid problems concerning planetary atmospheres and oceans can be described to some degree of approximation by this model. It uses the fact that, in a planet or frame in fast rotation, vertical velocities are small and slaved to the horizontal motion, so that flow patterns can be described in terms of two horizontal coordinates. The vertical depth of the fluid becomes a dependent variable. The fluid displays many of the unique properties of two-dimensional turbulence, but some of the aspects of three-dimensional dynamics are still important. In particular, topographic features of the bottom wall above which the fluid is flowing appear explicitly in the model. In terms of the streamfunction $\psi(\mathbf{x}, t)$, with $\mathbf{x} \equiv(x, y)$, the model reads:

$$
\frac{\partial \nabla^{2} \psi}{\partial t}+\lambda\left[\psi, \nabla^{2} \psi+h\right]=D+F .
$$

$D$ is the dissipation term. Here we will use the standard viscous damping, $D=\nu \nabla^{4} \psi$, where $\nu$ is the viscosity. $F(\mathbf{x}, t)$ is any kind of relative-vorticity external forcing, and $h=f \Delta H / H_{0}$,

${ }^{1}$ URL: http://www.imedea.uib.es/ 
with $f$ the Coriolis parameter, $H_{0}$ the mean depth, and $\Delta H(\mathbf{x})$ the local deviation from the mean depth. $\lambda$ is a bookkeeping parameter introduced to allow perturbative expansions in the interaction term. The physical case corresponds to $\lambda=1$. The Poisson bracket or Jacobian is defined as

$$
[A, B]=\frac{\partial A}{\partial x} \frac{\partial B}{\partial y}-\frac{\partial B}{\partial x} \frac{\partial A}{\partial y} .
$$

The streamfunction provides the horizontal components of the fluid velocity $(u(\mathbf{x}), v(\mathbf{x}))$ from

$$
u=-\frac{\partial \psi}{\partial y}, \quad v=\frac{\partial \psi}{\partial x}
$$

Equation (1) represents the time evolution of the relative vorticity subjected to forcing and dissipation. In addition to the context of rotating fluids, this kind of quasi-twodimensional dynamics appears also in the study of drift-wave turbulence in plasmas under strong magnetic fields (perpendicular to the plane of $\mathbf{x}$ ) $[4,5,6,7]$. In this case $\psi$ is related to the electrostatic potential, and $h=\ln \left(\omega_{c} / n_{0}\right)$, where $\omega_{c}$ and $n_{0}$ are the cyclotron frequency and plasma density respectively. Eq. (1) is also the limiting case of the more general Charney-Hasegawa-Mima equation when the scales are small compared to the ion Larmor radius or the barotropic Rossby radius $[5,6,7]$.

\section{The noise-induced currents}

The results in [2] imply (Rayleigh friction is used in that paper, but the results apply also to the viscous damping used here) that, when the forcing in Eq.(1) is a white Gaussian noise, the average flow at large scales approaches a state with currents following the large-scale features of the underlying topography. Energy is extracted from the noise forcing and concentrated into these large-scale currents. If noise is switched-off, viscosity dissipates all the energy in the system and currents stop. More interestingly, if nonlinear terms are eliminated from the equation, the average flow is again zero, corresponding to the intuitive idea that noise with zero average would not induce mean flow. But the interaction between noise and nonlinearity induces nonvanishing currents through the phenomenon of noise rectification. The direction of the currents was cyclonic (i.e. counterclockwise in the Northern hemisphere) around depressions and anticyclonic around elevations. An example of average flow induced by noise over the topography of Fig. 1 is shown in Fig. 2. It is obvious that contour levels of the average streamfunction, giving the average velocity field, closely follow topographic contours. Detailed inspection of the simulation data reveals that the correlation is higher for the large-scale features, the small scales being less correlated with topography.

Appearance of currents following topography is not a new issue. Since the work of Salmon et al. [8] it is known that the statistical mechanics of the Euler equations predicts such kind of currents. In addition, there seem to be observations of them in several places of the world ocean [9]. What is quite surprising and new is that they appear here in a forced and dissipative model, and without the property of detailed balance. Thus, the equilibrium statistical mechanics ideas 


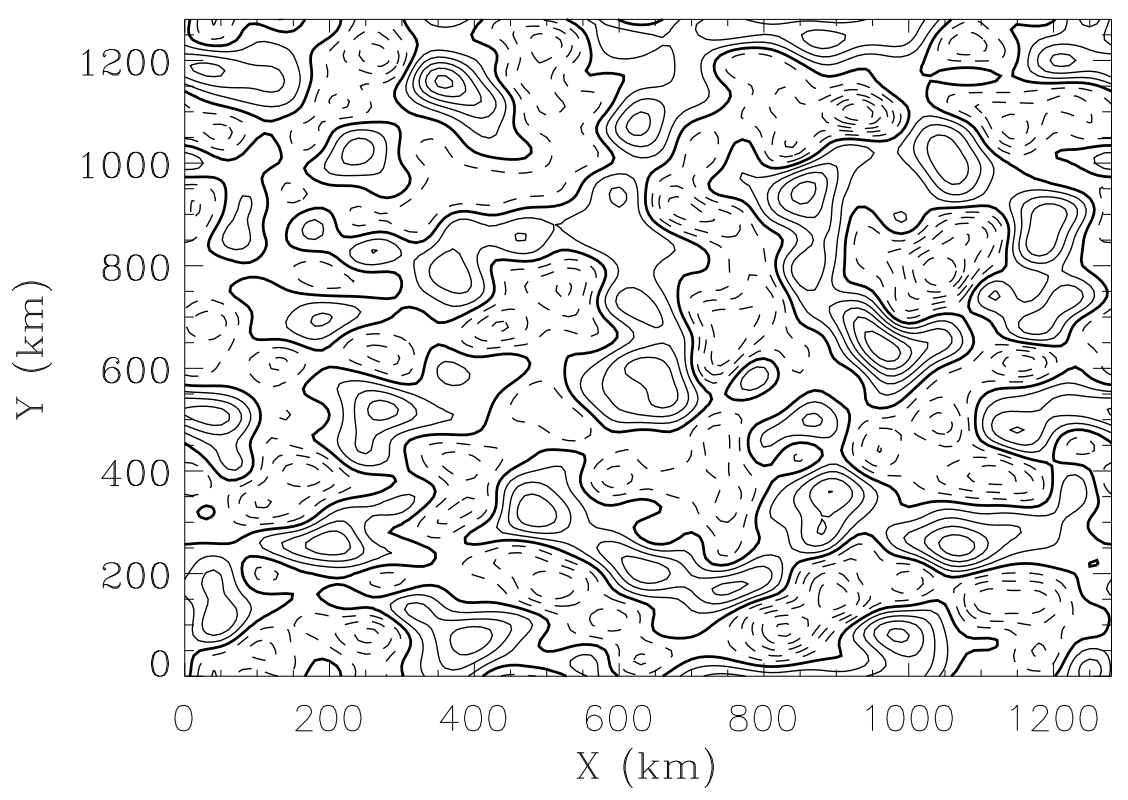

Figure 1: Depth contours of a randomly generated bottom topography. Maximum depth is $381.8 \mathrm{~m}$ and minimum depth $-381.8 \mathrm{~m}$ over an average depth of $5000 \mathrm{~m}$. Levels are plotted every $63.6 \mathrm{~m}$. Continuous contours are for positive deviations with respect to the mean, whereas dashed contours are for negative ones.

presented in [8] cannot explain the phenomenon in (1). A review of the different contexts where topographic currents appear can be found in [10].

In the next Section we perform analytic calculations trying to understand the origin of the currents in the present forced and dissipative case [11]. The observation of distinct behavior at different scales suggests that analysis of the relationship between small and large scales would give clues about the process.

\section{Coarse-graining approach}

Here we analyze how the dynamics of the large scales in (1) is affected by the small scales, when $F$ is a random forcing. A useful choice of $F$, flexible enough to model a variety of processes, is to assume $F$ to be Gaussian stochastic process with zero mean and correlations given by

$$
\left\langle\hat{F}_{\mathbf{k}}(\omega) \hat{F}_{\mathbf{k}^{\prime}}\left(\omega^{\prime}\right)\right\rangle=D k^{-y} \delta\left(\mathbf{k}+\mathbf{k}^{\prime}\right) \delta\left(\omega+\omega^{\prime}\right)
$$

$\hat{F}_{\mathbf{k}}(\omega)$ denotes the Fourier transform of $F(\mathbf{x}, t), \mathbf{k}=\left(k_{x}, k_{y}\right)$, and $k=|\mathbf{k}|$. The process is then white in time but has power-law correlations in space. $y=0$ corresponds to white-noise also in space, which is the case studied in [2] and displayed in Fig. 2. This value of $y$ has been observed for wind forcing on the Pacific ocean [12]. Thermal noise corresponds to $y=-4$ [13]. In this 


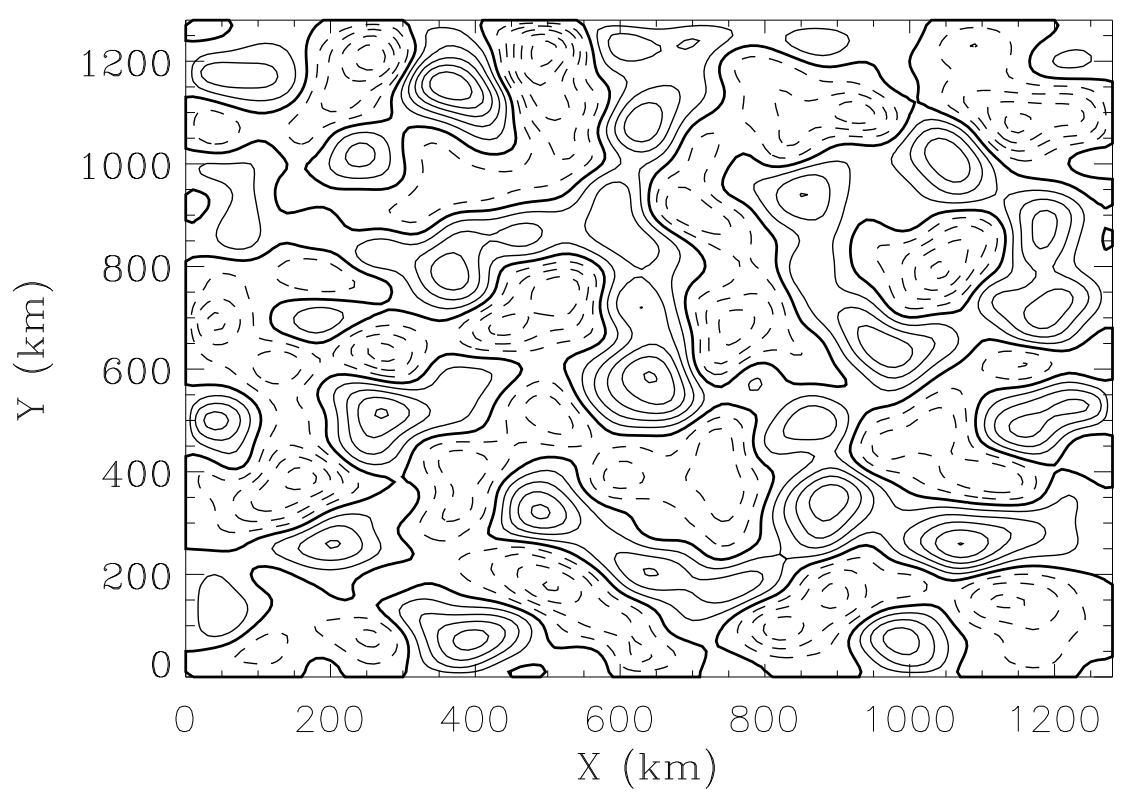

Figure 2: Mean streamfunction computed by time averaging when a statistically stationary state has been achieved. Continuous contours denote positive values of the streamfunction, whereas dashed contours denote negative ones; $\lambda=1, f=10^{-4} \mathrm{~s}^{-1}, \nu=200 \mathrm{~m}^{2} \mathrm{~s}^{-1}$, and forcing is white noise in space and time with intensity $D=10^{-9} \mathrm{~m}^{2} \mathrm{~s}^{-3}$. Maximum and minimum values are 991.864 and $-991.864 \mathrm{~m}^{2} / \mathrm{s}$, and levels are plotted every $165.31 \mathrm{~m}^{2} / \mathrm{s}$.

case there is a fluctuation-dissipation relation between noise and the viscosity term, so that the fluctuations satisfy detailed balance.

To obtain the desired large-scale closed equation we have applied a coarse-graining procedure to the investigation of the dynamics. For our problem it is convenient to use the Fourier components of the streamfunction $\hat{\psi}_{\mathbf{k} \omega}$ or equivalently the relative vorticity $\zeta_{\mathbf{k} \omega}=-k^{2} \hat{\psi}_{\mathbf{k} \omega}$. This variable satisfies:

$$
\begin{aligned}
\zeta_{\mathbf{k} \omega}= & G_{\mathbf{k} \omega}^{0} F_{\mathbf{k} \omega}+ \\
& \lambda G_{\mathbf{k} \omega}^{0} \sum_{\mathbf{p}, \mathbf{q}, \Omega, \Omega^{\prime}} A_{\mathbf{k p q}}\left(\zeta_{\mathbf{p} \Omega} \zeta_{\mathbf{q} \Omega^{\prime}}+\zeta_{\mathbf{p} \Omega} h_{\mathbf{q}}\right),
\end{aligned}
$$

where the interaction coefficient is:

$$
A_{\mathbf{k p q}}=\left(p_{x} q_{y}-p_{y} q_{x}\right) p^{-2} \delta_{\mathbf{k}, \mathbf{p}+\mathbf{q}}
$$

the bare propagator is:

$$
G_{\mathbf{k} \omega}^{0}=\left(-i \omega+\nu k^{2}\right)^{-1},
$$

and the sum is restricted by $\mathbf{k}=\mathbf{p}+\mathbf{q}$ and $\omega=\Omega+\Omega^{\prime} . \mathbf{p}=\left(p_{x}, p_{y}\right), p=|\mathbf{p}|$, and similar expressions hold for q. $0<k<k_{0}$, with $k_{0}$ an upper cut-off. Following the method in Ref. [14], one can eliminate the modes $\zeta_{k}^{>}$with $k$ in the shell $k_{0} e^{-\delta}<k<k_{0}$ and substitute their 
expressions into the equations for the remaining low-wavenumber modes $\zeta^{<}$with $0<k<k_{0} e^{-\delta}$. To second order in $\lambda$, the resulting equation of motion for the modes $\zeta^{<}$is:

$$
\frac{\partial \nabla^{2} \psi^{<}}{\partial t}+\lambda\left[\psi^{<}, \nabla^{2} \psi^{<}+h^{<}\right]=\nu^{\prime} \nabla^{4}\left(\psi^{<}-g h^{<}\right)+F^{\prime},
$$

where

$$
\begin{gathered}
\nu^{\prime}=\nu\left(1-\frac{\lambda^{2} S_{2} D(2+y) \delta}{32(2 \pi)^{2} \nu^{3}}\right), \\
g(\lambda, D, \delta, \nu, y)=\frac{\lambda^{2} D S_{2}(y+4) \delta}{16(2 \pi)^{2} \nu^{3}} .
\end{gathered}
$$

$F^{\prime}(\mathbf{x}, t)$ is an effective noise which turns out to be also a Gaussian process with mean value and correlations given by:

$$
\begin{gathered}
<F^{\prime}(\mathbf{x}, t)>=-\frac{\lambda^{2} D S_{2}(4+y) \delta}{16(2 \pi)^{2} \nu^{2}} \nabla^{4} h^{<}, \\
\left\langle( \hat { F } _ { k } ^ { \prime } ( \omega ) - \langle \hat { F } _ { k } ^ { \prime } ( \omega ) \rangle ) \left(\begin{array}{c}
\left.\left.\hat{F}_{k^{\prime}}^{\prime}\left(\omega^{\prime}\right)-\left\langle\hat{F}_{k^{\prime}}^{\prime}\left(\omega^{\prime}\right)\right\rangle\right)\right\rangle= \\
D k^{-y} \delta\left(k+k^{\prime}\right) \delta\left(\omega+\omega^{\prime}\right)
\end{array}\right.\right.
\end{gathered}
$$

$S_{2}$ is the length of the unit circle: $2 \pi$. Equations (8)-(12) give the dynamics of long wavelength modes $\psi^{<}$. They are valid for small $\lambda$ or, when $\lambda \approx 1$, for small width $\delta$ of the elimination band. The effects of the eliminated short wavelengths on these large scales are described in the new structure of the viscosity operator and the corrections to the noise term $F^{\prime}$. The action of the dressed viscosity term $\nabla^{4}\left(\psi^{<}-g h^{<}\right)$is no longer to drive large scale motion towards rest, but towards a motion state $\left(\approx g h^{<}\right)$characterized by the existence of flow following the isolevels of bottom perturbations $h^{<}$. This ground state would characterize the structure of the mean pattern. The energy in this ground state is determined by the function $g(\lambda, D, \delta, \nu, y)$ which measures the influence of the different terms of the dynamics (nonlinearity, noise, viscosity). Relation (10) shows that while nonlinearities and noise increase the energy level of the ground state, high values of the viscosity parameter would imply a reduction of the strength of the ground state motion due to the damping that viscosity exerts over small scales. The other mechanism that reinforces the existence of average directed motion comes from the fact that the dressed noise has got a mean component as a result of the small scale elimination.

A most interesting fact in (10) and (11) is the presence of the factor $y+4$. It implies that the tendency to form directed currents reverse sign as $y$ crosses the value -4 , and that it vanishes at $y=-4$ which is the value for thermal noise satisfying detailed balance. In consequence noise rectification does not occur when detailed balance holds, a result of general validity [1]. As an illustration we show in Fig. 3 the average streamfunction in a case with $y=-4$. An irregular flow is seen which does not follow the topographic contours. Increasing the number of configurations included in the average, the amplitude of the mean streamfunction structures is seen to decrease, converging towards zero flow. 


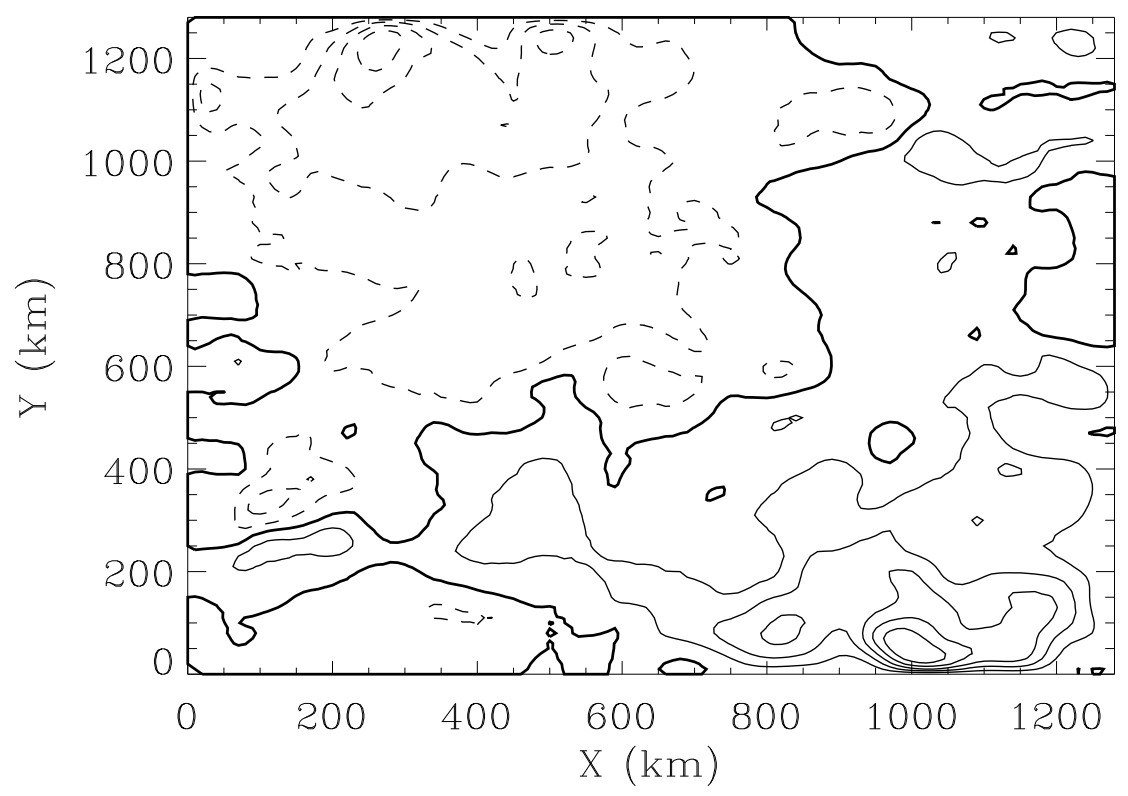

Figure 3: Mean streamfunction (average is over a period of 250 years) for the same parameters as in Fig. 2, except that noise correlations are of the form (4) with $y=-4$. Features are uncorrelated with topography. Averaging for longer times makes the features to disappear.

\section{Conclusions}

We have shown that quasigeostrophic flows develop mean patterns in the presence of noisy perturbations, and that the origin of these patterns is related with nonlinearity and lack of detailed balance. Nonlinear terms couple the dynamics of small scales with the large ones and provide a mechanism to transfer energy from the fluctuating component of the spectrum to the mean one. This mean spectral component, that is inexistent in purely two-dimensional turbulence [15], is controlled by the shape of the bottom boundary and characterizes the structure of the pattern. The existence of these noise-sustained structures has a wide range of implications in the fields of fluid and plasma physics. First because it highlights the important and organizing role that noise can play in these systems. Second, it establishes the need to modify not only the value of the parameters (as usually done in eddy-viscosity approaches) when performing large eddy simulations with insufficient small-scale resolution, but also the structure of the equations in a way determined by topography. This last statement has been previously suggested from a heuristic point of view in the context of large-scale ocean models $[9,10,16]$. Our results represent a step forward towards the justification of such approaches. The implications of these changes in presently existing general circulation ocean models open new ways for investigating the crucial role of oceanic circulation on climate variability. 


\section{Acknowledgements}

Financial support from FEDER and MCyT (Spain) (IMAGEN REN2001-0802-C02-01/MAR, CONOCE BFM2000-1108) is greatly acknowledged.

\section{References}

[1] M.O. Magnasco, Phys. Rev. Lett. 71, 1477 (1993); J. Maddox, Nature 369, 181 (1994); C. R. Doering, W. Horsthemke, J. Riodan, J. Phys. Rev. Lett. 72, 2984 (1994); J. Maddox, Nature 368, 287 (1994); J.K. Douglass, Lon Wilkens, Eleni Pantazelou, Frank Moss, Nature 365, 337 (1993); S. M. Bezrukov, Igor Vodyanoy, Nature 378, 362 (1995); J. Rousselet, L. Salome, A. Ajdari, J. Prost, Nature 370, 446 (1994).

[2] A. Álvarez, E. Hernández-García, J. Tintoré , Physica A 247, 312 (1997).

[3] J. Pedlosky , Geophysical fluid dynamics, Springer-Verlag (New York, 1987).

[4] A. Hasegawa, K. Mima , Phys. Fluids 21, 87 (1978).

[5] A. Hasegawa, C. G. Maclennan, Phys. Fluids 22, 11 (1979).

[6] A. Hasegawa, Advances in Physics 34, 1 (1985).

[7] W. Horton, Phys. Rep. 192, 1 (1990).

[8] R. Salmon, G. Holloway, M.C. Hendershot J. Fluid. Mech. 75, 691 (1976).

[9] G. Holloway , J. Phys. Oceanogr. 22, 1033 (1992).

[10] A. Álvarez and J. Tintoré, in Ocean Modeling and Parametrization, edited by E.P. Chassignet and J. Verron (Kluwer, Dordrecht, 1998).

[11] A. Álvarez, E. Hernández-García, J. Tintoré , Phys. Rev. E 58, 7279 (1998); ibid, Phys. Lett. A 261, 179 (1999); ibid., Geophys. Res. Lett., 27, 739 (2000).

[12] M.H. Freilich and D.B. Chelton , J. Phys. Oceanogr. 16, 741 (1985).

[13] L.D. Landau and M. Lifshitz, Fluid Dynamics, 2nd Edition. Course of Theoretical Physics, vol. 6 (Pergamon, New York, 1987).

[14] D. Foster, D. Nelson, M. Stephen, Phys. Rev. A 16, 732 (1977).

[15] P.D. Thompson, J. Fluid Mech. 55, 711 (1972).

[16] A. Álvarez, J. Tintoré, G. Holloway, M. Eby, J.M. Beckers , J. Geophys. Res., 99, 16053 (1994). 